\title{
NEW APPROACHES TO OPTIMIZING ANTIBACTERIAL THERAPY: PK/PD PARAMETERS
}

\author{
M. D. Zhelyazkova-Savova \\ Department of preclinival and clinical pharmacology, Varna Medical University
}

Reviewed by: Assoc. prof. N. Negrev, PhD

\begin{abstract}
Optimization of the antibacterial therapy constitutes an important clinical problem. A new possibility to solve it is to consider the pharmacokinetics (PK) and pharmacodynamics (PD) of these drugs in their unity. PK of antimicrobials has always been a significant factor in the drug choice, since it determines the accumulation of the drug at the site of infection. The current aspects of PD of antibacterial drugs, however, are only lately being intensively developed. Amongst the PD indices, only MIC (reflecting the susceptibility of the infective agent to drugs) has been routinely used in the clinical practice up to now. Currently, increasing attention is being paid to other PD parameters: pattern of bacterial killing (time- or concentration dependent) and persistent (post-antibiotic) effect. According to these parameters, antibacterial drugs can be classified into three groups. Combining the $P K$ indices maximal plasma concentration $\left(C_{\max }\right)$ and area under concentration-time curve (AUC) with PD ones (MIC) in a way based on experimental evidence, allows the development of integral PK/PD parameters, predicting clinical and efficacy and bacteriological cure. For beta-lactams and macrolides such a parameter is the time during which the concentration of the drug is higher than MIC $\left(\mathrm{T}>\mathrm{MIC}_{90}\right)$. With aminoglycosides and fluoroquinolones, the parameter correlating bets with positive outcomes, ate the ratios $\mathrm{C}_{\max } / \mathrm{MIC}_{90}$ and $\mathrm{AUC}_{24} / \mathrm{MIC}_{90}$. With azalides the best predictor of efficacy is $\mathrm{AUC}_{\mathbf{2 4}} / \mathrm{MIC}_{\mathbf{9 0}}$.

The introduction of PK/PG parameters in clinical performance has the potential to provide individual approaches in the choice of antibacterial drug and their dosing regimen. They can also contribute to reducing resistance and adverse effects, as well as to lowering the cost of therapy.
\end{abstract}

Keywords: antibacterial drugs, $\mathrm{PK} / \mathrm{PD}$ parameters, $\mathrm{C}_{\max } / \mathrm{MIC}_{90}, \mathrm{AUC}_{24} / \mathrm{MIC}_{90}, \mathrm{~T}>\mathrm{MIC}_{90}$

Antibacterial therapy aims at achieving optimal results in several aspects. It is the clinical response and infection eradication that are primarily sought, prevention of bacterial resistance is of no less importance. Therapy should also be carried out at the expense of minimal adverse effects and at the lowest cost.

The efficacy of the antibacterial therapy is determined by several factors. The choice of a drug and the dosing regimen are decisive for the positive outcome and for minimizing resistance.

The drug choice will depend on its own pharmacological characteristics. Additionally, the patient will determine the pharmacokinetic behavior of the drug, whilst the pathogen is important for its pharmacodynamics. Thus, a triad of factors is formed, where the elements DRUG, PATIENT and PATHOGEN all have their impact on the therapeutic process.

Address for correspondence:

M. D. Zhelyazkova-Savova, Dept. of preclinival and clinical pharmacology, Medical University Prof. Dr. Paraskev Stoyanov, 55 Marin Drinov St., BG-9002, Varna, BULGARIA

E-mail: mariadz52@yahoo.com
Pharmacokinetics (PK) of antimicrobial drugs is characterized by some specific features. Of these, the drug's distribution is essential for attaining therapeutic concentration at the site of infection. As a resultant variable of the kinetic processes in the organism (absorption, distribution, metabolism and excretion), the drug plasma concentration is central to pharmacokinetic studies. Its changes in time are defined by parameters such as Cmax, Tmax and $\mathrm{AUC}_{24}$ $\mathbf{C}_{\max }$, the concentration peak, reflects the extent of the absorption with various rotes of administration. Tmax measures the rate of absorption. $\mathbf{A} \mathbf{U C}_{24}$ is the area closed under the concentration-time curve and determines the amount of drug present in the organism during the corresponding period (e.g. for 24 hours).

Pharmacodynamics (PD) describes the effect of a drug as a function of its concentration. The development of PD of antibacterial drugs as a study of the drug's interaction with microbial cells has relatively lagged behind for a long time. Of the PD parameters, MIC (minimal inhibitory concentration) is the only one used routinely in daily practice. During the last two decades, however, there has been increasing interest to the PD of antimicrobial drugs. The development of the anti-infective pharmacology has lead to the accumu- 
lation of huge knowledge in this area. The knowledge has mainly concentrated on the pattern of antimicrobial killing and the persistent (post-antibiotic) effect, which, together with MIC, have been integrated in a new concept, combining in one the PD and PK of antibacterials to provide a better approach to successful therapy.

The pattern of antimicrobial killing refers to the dependence of the antibacterial action on concentration and time $(5,10)$. With some drugs, there is a proportional increase of the size and speed of bacterial growth inhibition with the increase of the drug concentration. These drugs are said to act in a concentration-dependent manner. This pattern is typical for the aminoglycosides, quinolones, ketolides. With other drugs, the dependence of killing on concentration is limited; these drugs are referred to as concentration-independent. Instead, the effect is increasing with prolongation of the bacteria exposure to the drug. This pattern of killing is known as time-dependent. It is characteristic of beta-lactams, macrolides, etc.

The post-antibiotic effect (PAE) is a well known in vitro phenomenon. It is defined as the persistent suppression of bacterial growth after limited exposure to the antibacterial drug (5). PAE reflects the time taken for the bacteria to resume their logarithmic growth. Most of the antimicrobials show in vitro PAE? 1.5 hours towards sensitive Gram positive cocci. However, towards Gram negative bacilli, only drugs affecting the protein or nucleic acid synthesis are capable of exerting PAE. Under the conditions of the living organism the PAE is usually much longer than in the laboratory. The sub-inhibitory antibacterial concentrations (sub-MIC) and the post-antibiotic leukocyte enhancement (PALE) are known to act as potentiating factors in vivo. It has been shown under experimental conditions that, when a bacterial culture has been pretreated with supra-MIC concentrations of an antibacterial drug, and then treated with sub-MIC concentrations, a more prolonged inhibition of bacterial growth results. The microorganisms can also be sensitized to the leukocyte phagocytosis by previous exposition to drug concentrations higher than MIC. Modern approaches aiming to optimize antibacterial therapy are based on experimental models of infections, where the treatment is based on both the PK and PD of drugs. PK and PD integration provides a better understanding of the development of the effect vs. time. The PK/PD concept allows improving the outcome of the antibacterial therapy; this has been proved experimentally and is now being confirmed by clinical trials. Specific PK/PD parameters have been determined, predicting success of the anti-infective therapy $(3,5,7)$.

From the standpoint of their PK/PD profile, the antibacterial drugs can be typified into 3 main categories $(1,12)$ :

1. Drugs of concentration-dependent action and pronounced PAE: aminoglycosides, fluoroquinolones, azalides, nitroimidazoles. The aim of the therapy will be producing a maximal concentration (that can be tolerated). The PK/PD predictor of efficacy will be $\mathbf{C m a x} / \mathbf{M I C}$ and $\mathrm{AUC}_{24} / \mathbf{M I C}$.
2. Drugs of time-dependent action and no or small PAE: beta-lactams, macrolides. The therapy objective with these drugs will be maximal duration of exposure. The parameter predicting success will be Time $>$ MIC.

3. Drugs of time-dependent action and moderate-toprominent PAE: azalides, tetracyclins, vancomycin. With these drugs, maximal drug amount in the organism is sought to attain and this is reflected by the parameter $\mathrm{AUC}_{24} / \mathbf{M I C}$.

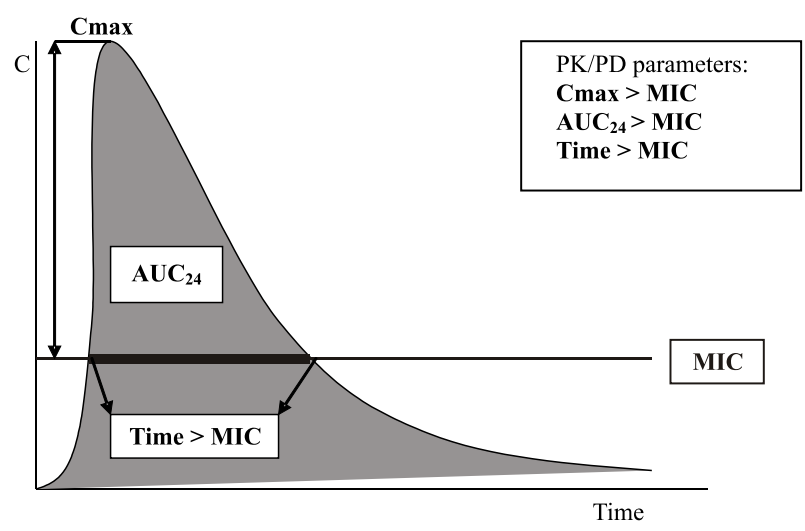

Fig.1. Pharmacokinetic/pharmacodynamic predictors of efficacy

The parameter $\mathbf{C m a x} / \mathbf{M I C}_{\mathbf{9 0}}$ is the ratio of the peak plasma concentration of the drug to the value of the $\mathrm{MIC}_{90}$, determined in the microbiological laboratory (fig.1). This ratio is the most important predictor of efficacy for the aminoglycosides. It has been found, that when $\mathrm{C}_{\max }$ of these drugs exceeds the value of $\mathrm{MIC}_{90}$ at least 8-10 times, there have been optimal results of the therapy, both in terms of microbiological cure and clinical response. Such an index can be achieved by giving the daily dose of the aminglycosides as a single, in stead of divided doses. In addition to efficacy, the safety of the therapy will also be improved. Giving the drug in extended dosing intervals allows attaining lower trough concentrations, which are known to correlate with lower toxicities. Finally, the single dose daily regimen is more cost-effective, sparing the expenses of multiple injections and stuff labor. This dosage regimen, although still debated, has been widely accepted and its advantages have been appreciated in the common practice $(2,5)$.

The parameter $\mathbf{A U C}_{\mathbf{2 4}} / \mathbf{M I C} \mathbf{C}_{\mathbf{9 0}}$ is the ratio of $\mathrm{AUC}_{24}$ and the $\mathrm{MIC}_{90}$ (fig. 1). It is similar to $\mathrm{C}_{\max } / \mathrm{MIC}_{90}$ in terms of its predictive value, but seems to be relatively more important for the fluoroquinolones (13). The threshold magnitude of this ratio has been a matter of debate. It turns out to depend on both the pathogen and the patient. With Gram positive infections and immunocompetent subjects a value of 30-40 appears to be sufficient. However, Gram negative infections and/or immunocompromised patients will require a value of at least 100-125 (6,10).

$\mathrm{Cmax} / \mathrm{MIC}_{90}$ is a similarly useful parameter with fluoroquinolones, but in addition to predicting efficacy, it 
can also alarm about emerging resistance. Values of less than 10 tend to be informative in this respect (16), although high $\mathrm{AUC}_{24} \mathrm{MIC}_{90}$ ratios have been shown to prevent resistance development $(7,18)$. On table 1 (modified from 16), three fluoroquinolones are presented, with their typical dosing and the parameters $\mathrm{AUC}_{24} / \mathrm{MIC}_{90}$ and $\mathrm{C}_{\max } / \mathrm{MIC}_{90}$ for S. Pneumoniae. Ciprofloxacin is a well known first-generation drug with predominant activity towards Gram negative microorganisms. Levofloxacin and moxifloxacin are second-generation agents, noted for their improved activity towards Gram positive cocci. From the table it can be seen that ciprofloxacin is not suitable for this infection because of unacceptably low values of both PK/PD parameters. The parameters for moxifloxacin are excellent in terms of predicting both efficacy and resistance. With levofloxacin, the situation is marginal: its $\mathrm{AUC}_{24} / \mathrm{MIC}_{90}$ value is good, but the low magnitude of $\mathrm{C}_{\max } / \mathrm{MIC}_{90}$ (less than 10) requires attention because of resistance risk.

Table 1. PK/PD parameters of fluoroquinolones for $S$. pneumoniae

\begin{tabular}{||l|c|c||}
\hline \hline Drug (daily dosing) & AUC24/MIC & Cmax/ $_{\mathbf{9 0}}$ \\
\hline $\begin{array}{l}\text { Ciprofloxacin } \\
(2 \times 750 \mathrm{mg})\end{array}$ & 12 & 1.2 \\
\hline $\begin{array}{l}\text { Levofloxacin } \\
(1 \times 500 \mathrm{mg})\end{array}$ & $12-48$ & $3-6(\mathrm{NB} !)$ \\
\hline $\begin{array}{l}\text { Moxifloxacin } \\
(1 \times 400 \mathrm{mg})\end{array}$ & $124-192$ & $12-18$ \\
\hline \hline
\end{tabular}

It is important to note that whenever concentration or AUC is meant, it is the concentration of the free drug that is relevant to the PK/PD concept. Only the unbound drug is pharmacologically active. Measurements of total drug concentration can yield conflicting results (7).

With the second group of drugs, the parameter that best correlates with efficacy, is the time during which the drug concentration is kept above the $\mathrm{MIC}_{90}$ (Time>MIC) (fig.1). Specially designed experiments with model infections have demonstrated, that with Gram positive pathogens the bacteriologic cure approaches its maximum with exposure lasting $40-50 \%$ of the dosing interval. With Gram negative bacteria, a longer exposure might be necessary, at least $70 \%$ of the dosing interval. Similarly, in immunocompromized patients, the dosing intervals should be almost entirely covered by the drug at concentrations 3-4 times higher than $\mathrm{MIC}_{90}$. This requirement will be best achieved by continuous drug infusion.

Table 2 (modified from 5) illustrates the PK/PD parameter predicting the efficacy of several parenteral and oral beta-lactams for $S$. Pneumoniae that is intermediately-sensitive or resistant to penicillin G, according to the corresponding values of MIC90. It can be seen, that even in this situation penicillin $\mathrm{G}$ can be used to manage the infection, if applied in sufficiently large doses. Amoxicillin will also be effective, as will the injectable ampicillin and ceftriaxon. However, cefaclor and cefuroxime at the specified doses will be completely or partly, respectively, ineffective.

Table 2. PK/PD parameters of three oral and three parenteral beta-lactams for $S$. pneumoniae with different susceptibility to penicillin $G$.

\begin{tabular}{||c|c|c|c|c||}
\hline \hline \multirow{2}{*}{$\begin{array}{c}\text { Drug } \\
\text { (daily dosing) }\end{array}$} & \multicolumn{2}{|c|}{$\mathrm{S}$. Pneumoniae (I) } & \multicolumn{2}{c||}{ S. Pneumoniae (R) } \\
\cline { 2 - 5 } & $\mathrm{MIC}_{90}$ & $\begin{array}{c}\mathrm{T}>\mathrm{MIC}_{90} \\
(\%)\end{array}$ & $\mathrm{MIC}_{90}$ & $\begin{array}{c}\mathrm{T}>\mathrm{MIC}_{90} \\
(\%)\end{array}$ \\
\hline $\begin{array}{c}\text { Amoxicillin } \\
(3 \times 1000 \mathrm{mg})\end{array}$ & 1 & 55 & 2 & 43 \\
\hline $\begin{array}{c}\text { Cefaclor } \\
(3 \times 1000 \mathrm{mg})\end{array}$ & 16 & 0 & 64 & 0 \\
\hline $\begin{array}{c}\text { Cefuroxim } \\
(2 \times 1000 \mathrm{mg})\end{array}$ & 2 & 40 & 8 & 0 \\
\hline $\begin{array}{c}\text { Penicillin G } \\
(4 \times 2 \mathrm{mln})\end{array}$ & 1 & 66 & 4 & 41 \\
\hline $\begin{array}{c}\text { Ampicillin } \\
(4 \times 1000 \mathrm{mg})\end{array}$ & 2 & 71 & 4 & 54 \\
\hline $\begin{array}{c}\text { Ceftriaxone } \\
(1 \times 1000 \mathrm{mg})\end{array}$ & 1 & 100 & 2 & 48 \\
\hline \hline
\end{tabular}

With azalides, tetracyclines and vancomycin, similar principles apply as with fluoroquinolones, the most appropriate parameter for assessment of their efficacy being the $\mathrm{AUC}_{24} / \mathrm{MIC}_{90}$. To predict positive outcome, this $\mathrm{PK} / \mathrm{PD}$ index is estimated to be no less than 100-125.

How is the PK/PD concept expected to work in practice? To aid its practical application, an approach has been suggested by developing PK/PD breakpoints based on the above indices $(9,10)$. These breakpoints refer to blood concentration of the drug that should be kept higher or at least equal to MIC. To determine the breakpoint magnitude, personal patient's data are utilized if the plasma concentration can be measured, or, alternatively, population kinetics information can be used. On the other hand, the local pathogen characteristics are taken into account, using the $\mathrm{MIC}_{90}$ of the particular infective agent. Combining these two variables will allow optimizing therapy in terms of adapting it to the characteristics of both the individual patient and pathogen.

Table 3. PK/PD breakpoints of fluoroquinolones for $S$. pneumoniae

\begin{tabular}{||l|c|c|c||}
\hline \multicolumn{1}{|c|}{ Drug } & Daily dosing & $\begin{array}{c}\text { PK/PD } \\
\text { Breakpoint }\end{array}$ & MIC $_{90}$ \\
\hline Ciprofloxacin & $2 \times 750 \mathrm{mg}$ & 1 & 2 \\
\hline Ofloxacin & $2 \times 400 \mathrm{mg}$ & 2 & 2 \\
\hline Levofloxacin & $1 \times 500 \mathrm{mg}$ & 2 & 1 \\
\hline Grepafloxacin & $1 \times 600 \mathrm{mg}$ & 0.5 & 0.25 \\
\hline \hline
\end{tabular}


For the drugs with concentration-dependent action, therefore, the breakpoint for a Gram-positive agent can be calculated by dividing the $\mathrm{AUC}_{24}$ by 30 . As seen from table 3 (modified from 10), the three fluoroquinolones at the doses shown, differ from each other in respect to their action on $S$. pneumoniae. In spite of the same $\mathrm{MIC}_{90}$ values, ciprofloxacin and ofloxacin will have different breakpoints, reflecting different pharmacokinetics, respectively, different plasma concentration and $\mathrm{AUC}_{24}$. The relationship between the $\mathrm{PK} / \mathrm{PD}$ breakpoints and $\mathrm{MIC}_{90}$ renders ciprofloxacin unsuitable for treating of this particular infection, whilst ofloxacin will be marginally effective. Levofloxacin demonstrates the best parameters in this case, the PK/PD breakpoint being higher than the $\mathrm{MIC}_{90}$ value. In the case of time-dependent antibacterials, the PK/PD breakpoint will be defined as the concentration that should be "covered" by the drugs during at least $40 \%$ of the dosing interval. Thus, amongst the beta-lactams shown in table 4 (modified from 10), the appropriate agents for the penicillin-resistant $S$. pneumoniae will be penicillin itself, amoxicillin and cefotaxim. However, cefuroxim PO and meropenem will be unsuitable.

Table 4. PK/PD breakpoints of beta-lactams for $S$. pneumoniae

\begin{tabular}{||l|c|c|c||}
\hline \multicolumn{1}{|c|}{ Drug } & Daily dosing & $\begin{array}{c}\text { PK/PD } \\
\text { Breakpoint }\end{array}$ & MIC90 \\
\hline Penicillin G & $4 \times 2 \mathrm{mln}$ UI & 4 & 4 \\
\hline Amoxicillin & $3 \times 500 \mathrm{mg}$ & 2 & 2 \\
& $2 \times 875 \mathrm{mg}$ & 2 & 2 \\
\hline Cefuroxim p.o. & $2 \times 500 \mathrm{mg}$ & 1 & 8 \\
\hline Cefotaxim & $3 \times 1000 \mathrm{mg}$ & 2 & 2 \\
\hline Meropenem & $3 \times 500 \mathrm{mg}$ & 1 & 2 \\
\hline \hline
\end{tabular}

The era of the clinical application of the PK/PD concept is only at its dawn. The practical experience is still poor. The expected positive results based on pre-clinical experiments are already beginning to happen. For now, the concept has been supported by positive results mainly in the treatment of respiratory tract infections such as community acquired pneumonia $(4,16,17)$. Evidence also exists of its successful application in the treatment of otitis media in children, orodental and urogenital infections, etc. The drugs most intensively studied are beta-lactams, macrolides $(14,17)$, fluoroquinolones $(15,16,18)$. Overall, the concept is applicable where the free drug concentration at the site of infection is in equilibrium with plasma concentration and the drug access is not limited by e.g. blood brain barrier. The initial results are promising and stimulating. Best outcomes have been demonstrated with severe infections and resistant pathogens. The optimal eradication of the causative agent prevents selection of resistant species. The pharmacoeconomic aspects are also favorable: shortening of the therapy and hospitalization duration reduces the treatment cost. Finally, the PK/PD concept encourages the development of new drug formulations with extended release, allowing not only improvement of the patients' compliance, but also the maintenance of the drug concentration at the desired level for longer periods of time $(8,12,17)$. In conclusion, the PK/PD concept is slowly being established in the clinical practice as an effective approach for solving the major problems facing the anti-infective therapy. With their practical relevance, it is certain that PK/PD principles will serve in the future the role of leading standards of antibacterial performance.

\section{REFERENCES}

1. $\mathrm{A} P K / \mathrm{PD}$ approach to antibiotic therapy. http:/www.rxkinetics.com/antibiotic_pk_pd.html

2. Barclay ML, KirkpatricK CM, Beqq EJ. Once daily aminoglycoside therapy. Is it less toxic than multiple daily doses and how should it be monitored? Clin Pharmacokinet 1999, 36(2):89-98

3. Barger A, Fuhst C, Wiedemann B. Pharmacological idices in antibiotic therapy. J Antimicrob Chemother 2003, 53:898-898

4. Calbo E., J. Garau. Application of PK and PD to antimicrobial therapy of community acquired respiratory tract infections. Respiration 2005, 72:561-571

5. Craig WA. Pharmacokinetic/pharmacodynamic parameters: Rationale for antibacterial dosing of mice and men. Clinical Infectious Diseases 1998, 26:1-12

6. Craig WA. Does the dose matter? Clinical Infectious Diseases 2001, Suppl. 3: S233-7

7. Drusano GL. Antimicrobial pharmacodynamics: critical interactions of 'Bug and Drug'. Nature reviews 2004, 2:289-300

8. Finch RG. Standards of antibacterial performance. Clin Microbiol Infect 2004, 10 (Suppl. 2): 1-5

9. Jacobs MR. Optimisation of antimicrobial therapy using pharmacokinetic and pharmacodynamic parameters. Clin Microbiol Inf 2001, 7: 589-596

10. Jacobs MR. Pharmacodynamic approach to antimicrobial treatment for respiratory infections. $J$ Microbiol Infect 2001, 7(11): 589-596

11. Jacobs MR. How can we predict bacterial eradication? Int J Infect Dis. 2003, Suppl 1:S13-20.

12. Jacobs MR. Building in efficacy: developing solutions to combat drug-resistant S. pneumoniae. Clin Microbiol Infect 2004, 10 (Suppl. 2): 18-27

13. Nix DE. Optimization of antimicrobial therapy to minimize medication errors. Medscape Pharmacists 2002, 3(1)

14. Peric M, Browne FA, Jacobs MR, Appelbaum PC. Activity of nine oral agents against gram-positive and gram-negative bacteria encountered in community-acquired infections: use of pharmacokinetic/ pharmacodynamic breakpoints in the comparative assessment of beta-lactam and macrolide antimicrobial agents. Clin Ther. 2003, 25(1):169-77.

15. Preston SL et a1. Pharmacodynamics of levofloxacin. JAMA 1998, 279(9):125-129

16. Suh B. Pharmacokinetics and pharmacodynamics of fluoroquinolones in respiratory tract infections. 
New approaches to optimizing antibacterial therapy: PK/PD parameters

http://scholar.google.com/url?sa=U\&q=http://www.an sorp.org/isaar/2001/blue/40.pdf

17. White A. R. et al. Augmentin in the treatment of community acquired respiratory tract infections: a review of the continuing development of an innovative antimicrobial agent. J Antimicrob Chemother 2004, 53, suppl. SI, i3-i20

18. Wispelwey B. Clinical implications of pharmacokinetics and pharmacodynamics of fluoroquinolones. Clinical Infectious Diseases 2005, 41:S127-135 